\title{
VACUUM PERFORMANCE AND BEAM LIFE TIME IN THE PEP II STORAGE RINGS*.
}

\author{
U. Wienands, SLAC, Stanford, CA 94309, USA
}

\begin{abstract}
The vacuum systems of the storage rings of the PEP-II $B$-Factory have had by now over two years of production running at high beam current and seen synchrotron radiation from almost $4 \mathrm{kAh}$ (High Energy Ring) and $10 \mathrm{kAh}$ (Low Energy Ring) of integrated beam current. The systems have performed well, reaching the design pressures and being able to cope with beam currents of almost $1 \mathrm{~A}$ (HER) and in excess of $2 \mathrm{~A}$ (LER). The photo-desorption coefficient $\eta$ was found in the HER to have reached values as low as $10^{-7}$. The shielded bellows have performed very well, with only one pair of bellows assemblies damaged due to higher-order mode generation from a known, nearby source. Issues encountered during normal operation have been a significant amount of dust trapping in the HER and the effect of a cloud of photo-electrons around the positron beam affecting the LER beam size. A couple of chambers in the HER failed; these failures arose from a combination of design and fabrications issues. The beam life time in the LER has been lower than expected based on the vacuum pressure and has been determined to be Touschek limited.
\end{abstract}

\section{INTRODUCTION}

The PEP-II storage rings[1] have six straight sections and six arcs. In the 9-GeV High Energy Ring (HER), the arc vacuum chambers are made of copper, the outer wall of which essentially forms a long photon dump capable of dissipating $100 \mathrm{~W} / \mathrm{cm}$ linear power density, sufficient for $3 \mathrm{~A}$ of beam current[2]. A cross section is shown in Fig.1. The Low Energy Ring (LER) arcs use aluminum extrusions

Figure 1: Arc chamber cross section for the HER

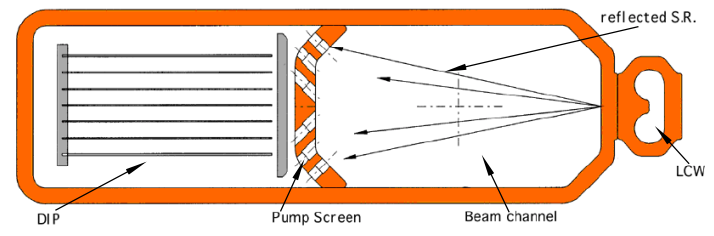

(Fig.2) with antechambers and discrete photon stops capable of absorbing $15 \mathrm{~kW}$ of radiation power each, again designed for $3 \mathrm{~A}$ of LER beam current[3]. In the LER, therefore only small areas of the vacuum chamber are hit by synchrotron radiation, which shows itself in a reduced rate of cleaning up ("scrubbing"). Pumping is done mostly

\footnotetext{
${ }^{*}$ Supported by DOE under contract DE-AC03-76SF00515
}

Figure 2: Arc chamber cross section for the LER

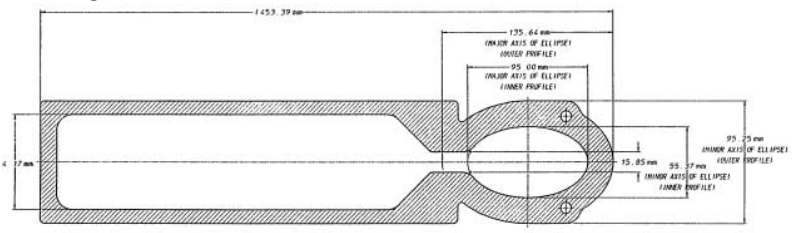

by distributed ion pumps (DIPs) with $120 \mathrm{l} / \mathrm{s} / \mathrm{m}$ pumping speed (incl. screen conductance) in the HER arcs and with $2000 \mathrm{l} / \mathrm{s}$ titanium sublimator pumps (TSPs) at the LER photon stops. In both cases, small ion pumps of 60 to $200 \mathrm{l} / \mathrm{s}$ speed serve as holding pumps when the main pumps are off (or, in case of the LER, before flashing the TSPs).

The straight-section vacuum system for both rings is made of round, seamless stainless steel (316L) tubing[4]. Two concentric tubes are used with cooling water in between. The linear heat load is moderate, less than $7 \mathrm{~W} / \mathrm{cm}$. In the quadrupole magnets, not enough room was available for the double-walled pipe and therefore single tubing is used with brazed-on cooling lines and a $1 \mathrm{~mm}$ outside copper cladding to distribute the heat load. Table 1 summarizes the parameters of both systems.

Table 1: PEP-II vacuum system parameters

\begin{tabular}{lcc} 
Parameter & LER & HER \\
\hline Beam current & $2.14 \mathrm{~A}$ & $0.95 \mathrm{~A}$ \\
Total rad. power & $1.3 \mathrm{MW}$ & $3.5 \mathrm{MW}$ \\
Power density & $6.8 \mathrm{~kW} / \gamma$-stop & $35 \mathrm{~kW} / \mathrm{m}$ \\
Pumping in arcs & $2000 \mathrm{1} / \mathrm{s} / \mathrm{stop} \mathrm{TSP}$ & $1201 / \mathrm{s} / \mathrm{m} \mathrm{DIP}$ \\
Pumping, straights & $19 \times 400 \mathrm{1} / \mathrm{s}$ & $19 \times 400 \mathrm{l} / \mathrm{s}$ \\
Arc pressure at 3 A & $<3 \mathrm{nTorr}$ & $<3 \mathrm{nTorr}$ \\
Straight press., 3 A & $<10 \mathrm{nTorr}$ & $<10 \mathrm{nTorr}$ \\
IR2 pressure at 3 A & $<1$ nTorr & $<1$ nTorr \\
\hline
\end{tabular}

\section{PRESSURE AND SYSTEM CLEANING}

After more than a year of production running, the LER has accumulated almost $10000 \mathrm{Ah}$ of integrated beam current while the HER has accumulated about $4000 \mathrm{Ah}$. The average base pressure is now below $1 \mathrm{nTorr}$, except for some identified or suspected leaks. The pressure differentials in the LER (Fig. 3) is less than $1 \mathrm{nTorr} / \mathrm{A}$, therefore at $3 \mathrm{~A}$ beam current the average epressure in the arcs will be about 4 nTorr, better than the design criterion of 10 nTorr. The pressure-differential history for a represen- 
Figure 3: Pressure differentials in the LER, after $10 \mathrm{kAh}$

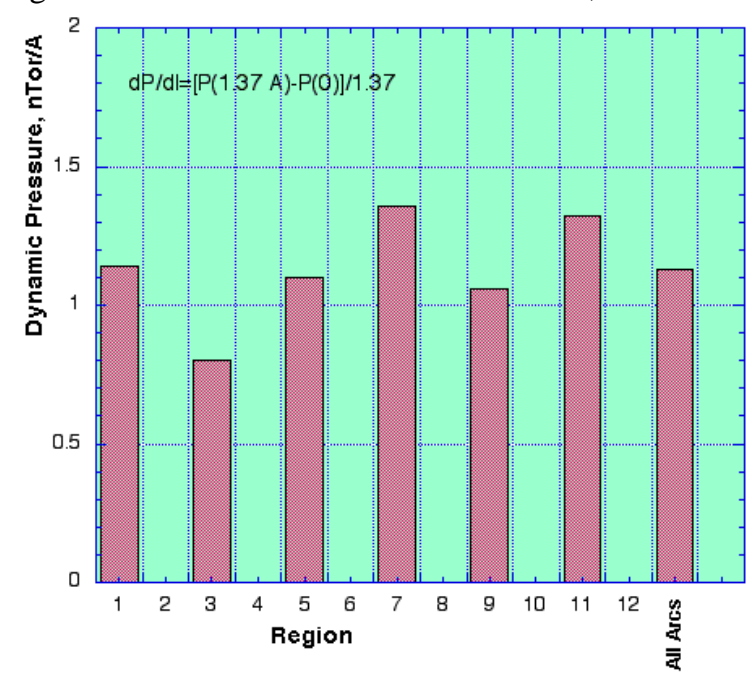

tative ion pump is shown in Fig. 4, showing that for the first $500 \mathrm{Ah}$ the pressure differential has come down faster than $1 /(I \times t)$. Since then, scrubbing has been considerably slower. A possible reason may be that the areas directly hit by radiation were at that point sufficiently clean that they no longer contribute to the total pressure. This leaves other areas not directly hit by radiation-which clean up considerably slower-to dominate the pressure. The HER base pressure is somewhat lower than the LER's, about 0.5 nTorr on average. Pressure differentials are about $0.3 \mathrm{nTorr} / \mathrm{A}$ on average. The history of the pressure differential $v s$ integrated beam current is shown in Fig. 5, for a representative distributed ion pump. Scrubbing has proceeded almost proportional to $1 /(I \times t)$ beyond $2000 \mathrm{Ah}$ integrated beam current. In fact, there is no hard evidence that scrubbing has slowed down. Since the speed of the DIPs in the HER arcs is known quite precisely from laboratory calibrations done at LLNL[5], we can calibrate the vertical axis in terms

Figure 4: LER pressure differential vs integrated dose

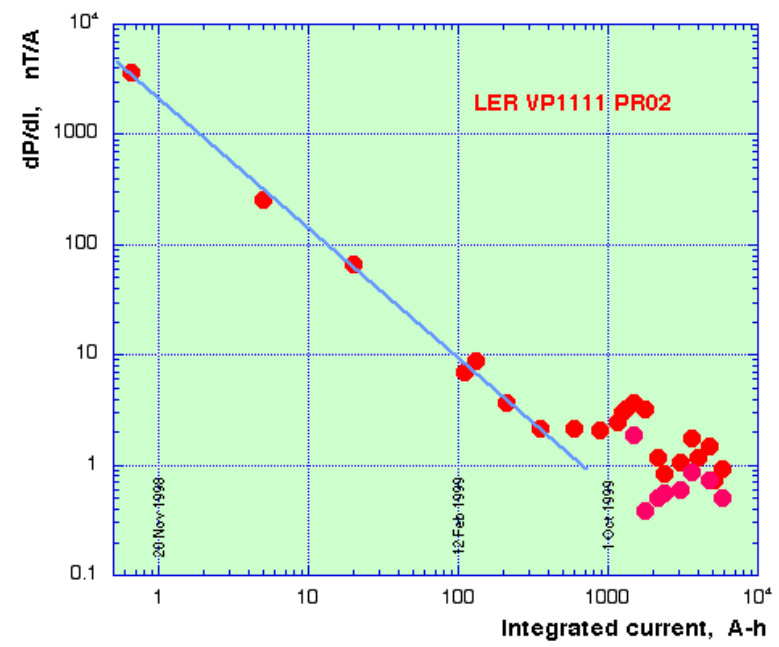

of the photon desorption coefficient $\eta$ using the formula:

$$
P=\frac{C}{L} \frac{L}{S} E I \eta
$$

where $L$ is the total arc length, $S$, the pump speed in $1 / \mathrm{s} / \mathrm{m}$ and $C=8.08 \times 10^{20}$ photons $/ \mathrm{GeV} / \mathrm{A} / \mathrm{s}$, a constant. In the PEP-II CDR[6], a value for $\eta$ of $2 \times 10^{-6}$ molecules/photon after $100 \mathrm{Ah}$ was used in calculating the expected pressures. This value was based on measurements taken at NSLS[7]. This reference point is indicated in Fig. 5, the performance of the vacuum system has come surprisingly close to the predictions. In fact, in both rings beam lifetime from gas scattering is no longer a relevant factor during normal operation.

Figure 5: HER pressure differential $v s$ integrated dose

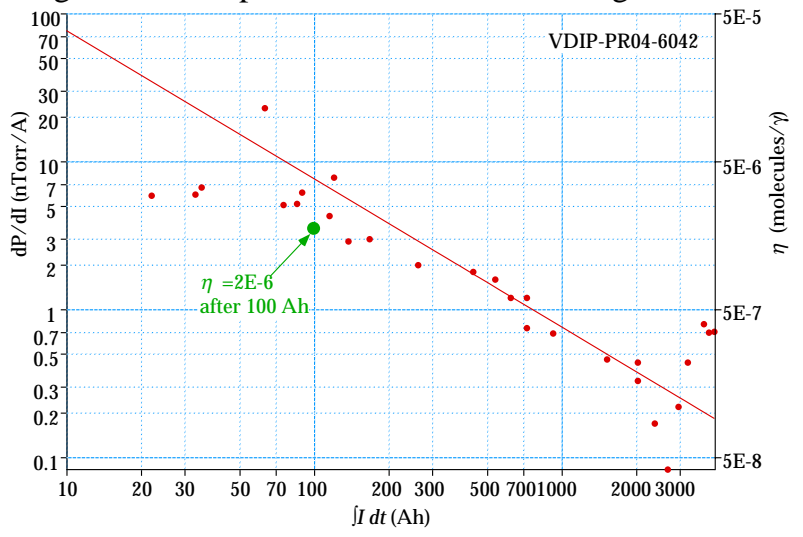

Vacuum pressures are monitored principally by the ionpump current. While this works well in the arc sections of the rings, large pressure differentials with beam current were encountered in the straight sections and in the HER arc holding pumps. The explanation for these large differentials was found in the time-constant of the pressure change on dropping the beam. While the current of the HER DIPs and the LER arc holding pumps drops with a measurable time constant of several minutes-consistent with the pumps' clearing away the desorbed gas load - the current of the straight-section pumps and of the HER arc holding ion pumps drops to a small value almost instantaneously, see Fig. 6. This indicates that the the pump current in these cases is not due to gas pressure; in fact, we suspected it to arise from photo-electrons, which was confirmed when it was shown that longitudinal magnetic fields in the pump tees and their vicinity could suppress most of the pump current.

\section{THERMAL MANGEMENT}

In several electron-beam facilities, the rf shields used to screen bellows and other components from beam-induced electromagnetic fields have been a source of difficulty esp. at high bunch currents. At PEP-II, we strieved to learn from these experiences as much as possible and a corresponding amount of time was spent during the engineering and design phase of the shielded bellows. Operationally, while 
Figure 6: Time dependence of ion-pump currents in the LER arc and straight sections

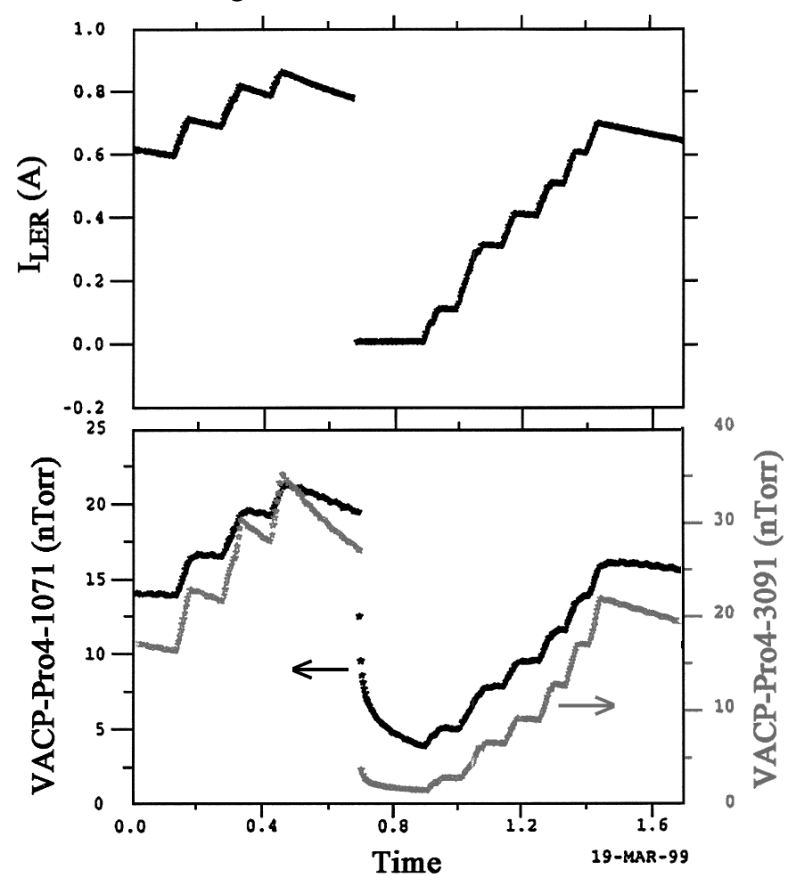

most of the bellows do show a moderate temperature rise proportional to $I^{2}$, a few showed temperature rises of up to $60^{\circ} \mathrm{F}$, reacing our limit at $150^{\circ} \mathrm{F}$. The actual power dissipated in the bellows at these temperature rises is quite small, on the order of $10 \mathrm{~W}$, but cooling by convection is suppressed due to protective layers of aluminum foil and an aluminum cover. We retrofit bellows with covers incorporating small muffin fans on an as-needed basis; even though the fans are powered electrically in series \& run slowly the small airflow is more than sufficient to suppress the temperature rise. The heating of only selected bellows has been traced to a resonant mode in the cavity between the rf shield and the bellows corrugations, which is of fairly narrow bandwidth. The set of bellows heating up changes with the bunch pattern in the ring.

No damage to bellows has been observed to date, with two exceptions. The rf shields of two bellows next to experimental beam collimators were damaged. These collimators were not tapered, but 0.6 in thick copper "half-moon" absorbers protruding into the vacuum chamber, and strong mode-conversion was to be expected. The bellows corrugations did not heat up significantly during operation and the damage was found only during replacement of the experimental collimators by fully engineered, tapered units. The nature of the damage of the fingers (Fig. 7) indicates heating of the shield fingers, although there is also narrowing of some fingers (at about 2 oclock in Fig. 7) which could indicate electric discharges in that area of the rf shield.

One component of concern is the $\mathrm{BeO}$ vertex chamber in the $\mathrm{BaBar}$ detector, which shows significant heating at one end. The heating appears to be due to higher-order modes and tracks $\hat{I} \times \bar{I}$ fairly well. From HER-LER interference measurements we deduce a wideband impedance centered broadly about $5.4 \mathrm{GHz}$ as being responsible for this heating effect. Simulations with MAFIA indicate the major source for these HOMs to be in the " $Y$ ", or crotch, area where the HER and LER vacuum systems meet[8].

Figure 7: Bellows assy. with damaged rf-shield fingers

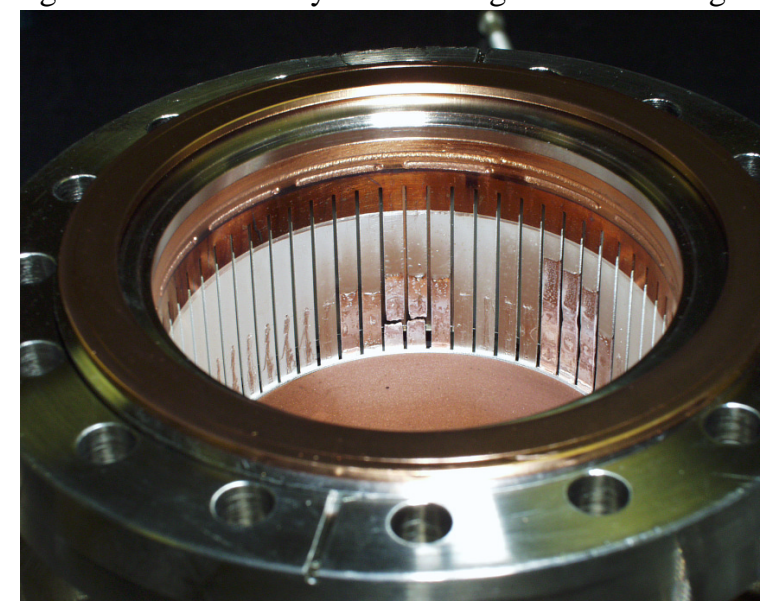

\section{DUST TRAPPING IN THE HER}

At the beginning of the year-2000 run the HER beam caused frequent background spikes in the BaBar detector, sometimes accompanied by a drop in beam lifetime. Dust trapping was suspected and confirmed when it was found that these events could be provoked by mechanically knocking on the vacuum chamber of the HER upstream of the detector using a remotely powered solenoid ("thumper"). To get the anatomy of such events, a fast (1 $\mathrm{kHz}$ ) data logger was built, reading out several scintillation counters placed close to the vacuum chamber for this purpose and also the current of nearby ion pumps. Fig. 8 shows such a recorded event, which begins by a series of sharp background spikes accompanied by a slight rise of current in a nearby ion pump (not seen on every event). The counter signal then indicates elevated beam loss rate until the beam is dumped by the radiation protection system. Another indication that the events are indeed local losses due to some scattering mechanism arises from the distribution of radiation in the BaBar protection diodes. This distribution agrees very well with the distribution of radiation from background caused by deliberately turning off certain ion pumps upstream of the detector in the HER as shown in Fig. 9.

The duration of these events (when they do not trigger the beam-abort system) varies from seconds to many minutes. Techniques tried to shorten the duration include shaking the beam using the injection system or excitation of mm-scale betatron oscillations using swept-frequency drivers. We have tried to reduce the backgrounds by taking the beams out of collision and by varying the angle of the HER beam at the IP. The success rate of these attempts has been low, typically $20 \%$. Reducing the beam current 
Figure 8: A background event in the fast data logger
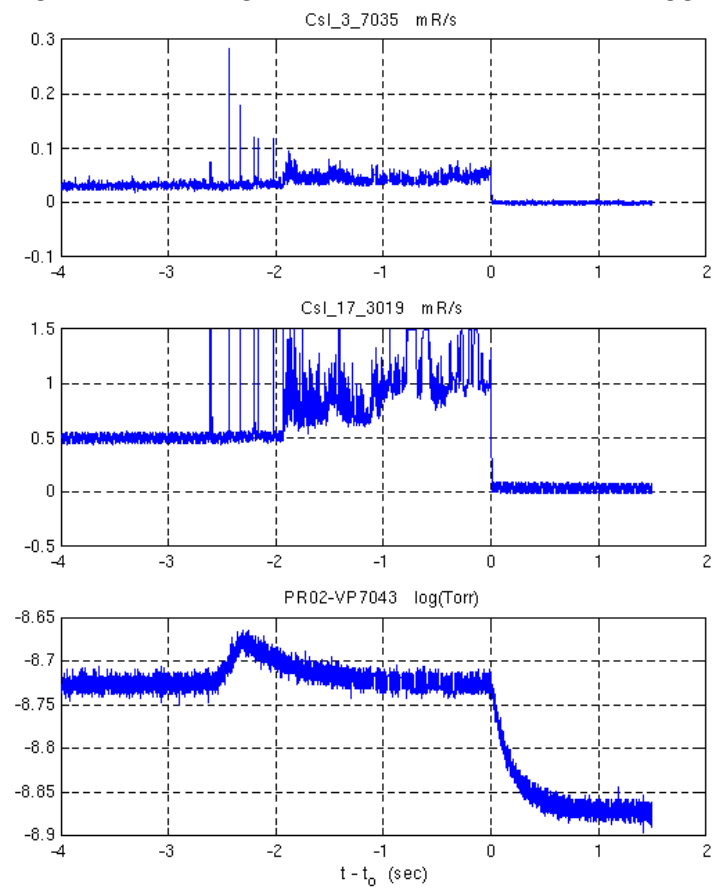

helps in reducing the frequency of these events as well as the amount of background radiation generated, however, both are affected in proportion to the beam current only, i.e. no threshold beam current below which dust-trapping no longer occurs has been observed. With increased beam exposure of the vacuum system however, this effect has subsided in frequency to the point where it is no longer

Figure 9: Radiation-intensity pattern from dust event (red dots) and pressure bump upstream in IR 2 (solid \& dashed lines).

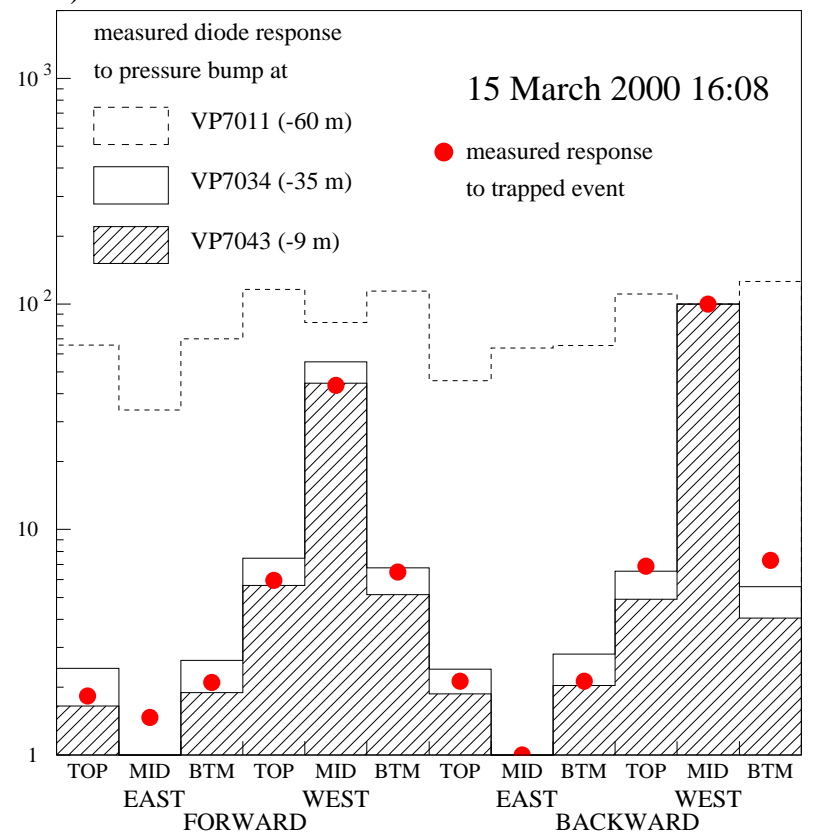

a major operational concern.

\section{ELECTRON CLOUD EFFECTS IN THE LER}

Once higher beam currents were achieved in the LER, strong nonlinear pressure rise was observed in the straight sections. This rise in ion pump currents could be reduced by solenoidal fields in the vacuum chamber of about $30 \mathrm{G}$ and was affected by the bunch pattern. For some

Figure 10: Multipactoring in the LER vacuum system

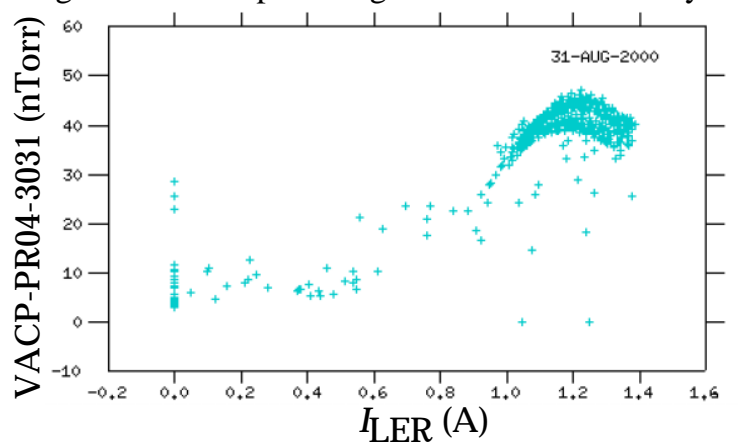

pumps, the current would actually go through a maximum and come down again with increasing beam current, see Fig.10. Coincident with the pressure rise, a growth in beam size was observed on the synchrotron-light monitor, which could also be mitigated by solenoidal fields in the beam pipe in the straight sections. All this is consistent with the presence of multipactoring electrons in the beam pipe. All LER straight sections have been fitted with solenoids, and the luminosity gain from these has been impressive, about $20 \%$ for the first two straight sections (of six) wrapped with solenoids. We have begun winding solenoids in the arcs as well, but due to a rigorous luminosity-delivery schedule this activity is proceeding only slowly[9].

\section{BEAM LIFETIME IN THE LER}

Even though the vacuum pressures in the LER have been actually quite low, the LER beam lifetime has always been perceived to be short. This prompted an investigation of the source of the beam life time limit in the LER. At a fixed total beam current we measured the beam life time $v$ s the total rf voltage in the ring for different bunch current. The result, shown in Fig. 11 together with estimates of Touschek and quantum life times, clearly show that at high bunch current the Touschek life time dominates.

\section{COMPONENT FAILURES}

Aside from the bellows rf shields mentioned above, two failures were encountered, both in the HER. In the first case, a leak occurred at a synchrotron mask in the interaction straight section upstream of the detector. This mask, made of copper, was brazed onto a stainless-steel 
Figure 11: Beam life time in the LER for various bunch currents
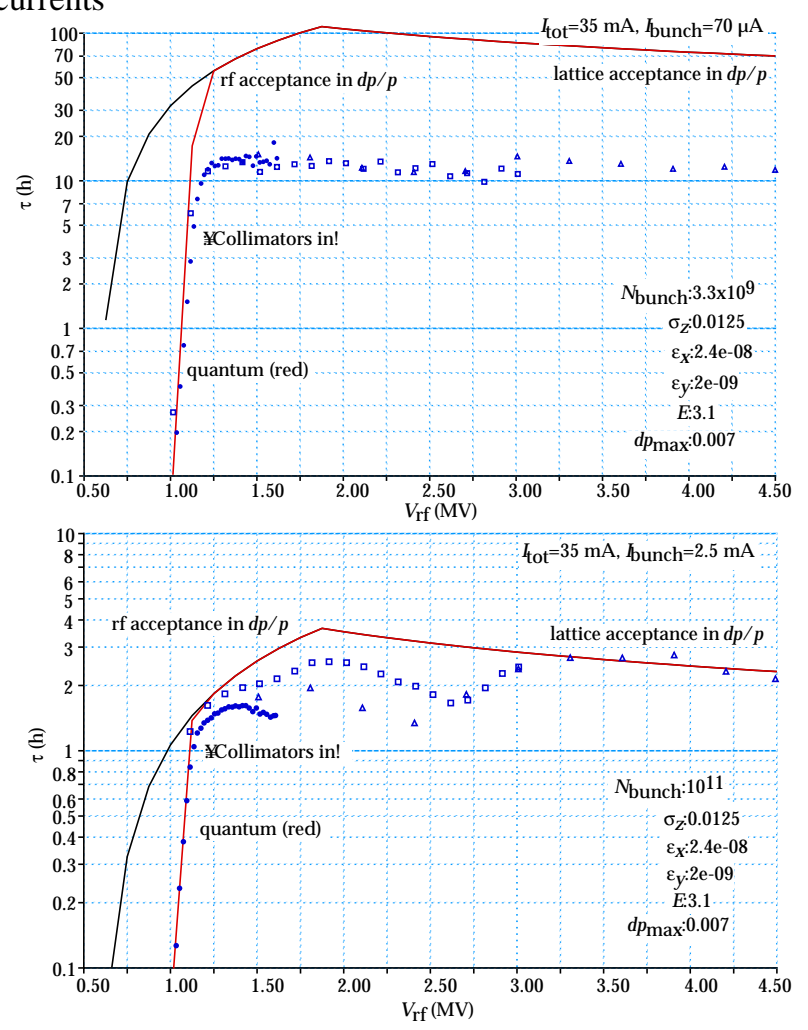

carrier plate which in turn was welded into a cut-out in the stainless steel vacuum chamber. Cooling lines were skipwelded on the outside of the carried plate. As it turned out, the cooling was insufficient, and mask and carrier were subject to considerable heating (the exact degree not being known but suspected to be in excess of $200^{\circ} \mathrm{F}$ ) at high beam current. Eventually, the weld seam of the carrier to the vacuum chamber failed. The chamber was rebuilt with the mask now being a solid copper, LCW-cooled, piece of chamber brazed to the end of the steel tube thereby reducing peak temperatures and stresses considerably.

The second failure was encountered in the so-called high-power dump chamber absorbing the radiation from the HER beam in the beam-separating magnets in the detector. This chamber is made of $\mathrm{Cu}$ plates welded together to make the chamber, with large flanges Cusil welded to an adapter piece at the end. In this case, the origin of the failure stems from the differential heating of the chamber, which was cooled on the side where the radiation strikes but not on the opposite side. Despite the lack of direct radiation hitting, the opposite side did heat up and thus the chamber wanted to bend. This it was restrained from doing by supports attached to the flanges, and the resulting stress led to failure of the Cusil joint. A new chamber was built with a redesigned joint (now a true full-penetration electron-beam weld), and also the cooling was modified to ensure much more uniform temperature of the chamber for all beam currents.

\section{SUMMARY}

The performance of the vacuum system of the PEP rings has been impressive and contributed to the speedy commissioning and reaching design luminosity of the $B$ Factory. Pressures and scrubbing have proceeded fairly closely along the predicted path, and beam-gas scattering no longer dominates the beam life time. At least in the HER photon-desorption coefficients as low as $10^{-7}$ have been seen.

\section{ACKNOWLEDGMENTS}

The author is indebted to the PEP team of accelerator physicists, engineers and and operators. In particular, A. Kulikov first recognized the ion-pump current as being dominated by photo electrons, managed the solenoid winding in the LER, and he provided many of the graphs in this paper. S. Ecklund together with A. Fisher and J. Vav'ra took a leading role in building the fast data logger and the analysis of the dust-trapping data. T. Schietinger (BaBar Collab.) provided Fig. 9. J. Seeman recognized the importance of the electron-cloud effect in the LER and launched the solenoid-winding project. The beam-lifetime experiment in the LER was carried out in collaboration with C. Steier and M. Zisman, LBNL. The effect of the solenoids on the LER beam size was studied experimentally by M. Minty (now at DESY), W. Kozanecki et al.

\section{REFERENCES}

[1] J.T. Seeman, these proceedings.

[2] C. Perkins et al., Vacuum System Design for the PEP-II B-Factory High Energy Ring, Proc. European Part. Accel. Conf., London, UK, 1994.

[3] D. Hunt et al., Design of the PEP-II Low Energy Ring Vacuum System, Proc. US. Part. Accel. Conf. Dallas, Tx, 1995, p. 2067.

[4] U. Wienands et al., The Vacuum System for the PEP-II High Energy Ring Straight Sections, ibid., p. 2129.

[5] F. Holdener et al., Test Results of Pre-Production Prototype Distributed Ion Pump Design for the PEP-II Asymmetric BFactory Collider. ibid., p. 2064.

[6] M. Zisman, PEP-II: An asymmetric B-Factory, Report SLAC-418, LBL-PUB-5379, June, 1993.

[7] ibid., p. 308ff.

[8] S. Ecklund et al.. these proceedings.

[9] A. Kulikov, et al., these proceedings. 